\title{
Stochasticity in tree growth closes the chain of evidence in forest structural dynamics
}

\author{
Jian Zhou ${ }^{1}$ \\ ${ }^{1}$ College of Urban and Environmental Sciences, Peking University
}

April 28, 2020

\begin{abstract}
Forest structural dynamics is governed by tree growth and mortality; While the mean growth rate has been integrated in mathematical derivation of forest size structure, variance in growth is always ignored. Here by solving Kolmogorov forward equation, I show the significant difference in forest size structure predictions with and without considering of the variance, which just solved the puzzle of mismatch between observation and theoretical prediction in previous studies. An asymptotically power-law relationship between tree size and growth rate variance was found and statistically interpreted, which is the key link in connecting all the evidences in forest size structure formation.
\end{abstract}

\section{Introduction}

Understanding the formation of forest size structure (i.e. the size-frequency distribution of trees) is crucial for forest protection and management. Although a great deal of statistics and theoretical investigations have been made, I would say that an essential defect in this research area results from the disadvantages in mathematical analysis, rather than lack of revealing new biological or ecological processes.

Two general governing equations, the McKendrick-von Foerster equation (Eq. 1) and the Kolmogorov forward equation (Eq. 2), have long been proposed for the size structure dynamics in plant communities(Von Foerster 1959; Hara 1984; Kohyama 1991; Condit \& Sukumar 1998; Coomeset al. 2003; Muller-Landau et al. 2006b), in which the former is commonly used, it can be considered as a simplification of the latter, by ignoring the variance of tree growth rate in the same size-class, i.e. letting $V(D)$ in Eq. 2 equals zero.

(1) (2) where $N$ represents individual number with size $D$ (measured by trunk diameter) and at time $t$, $G(D), V(D)$, and $M(D)$ are mean growth rate, growth rate variance, and mortality in each size-class, respectively.

Although growth rate variance is inevitable in natural world, the reason for rarely using Kolmogorov forward equation is largely due to difficulties in solving the second-order partial differential equation analytically, and ecologists believe that discarding the variance term has little impact in analyzing forest size structure under demographic equilibrium state, so most studies are based on the equilibrium solution of McKendrick-von Foerster equation $N(D)=N_{0} \exp \left[-[?] M(D) G(D)^{-1} d D\right] G(D)^{-1}$ (where $N_{0}$ is a constant representing the ideal number of trees in the smallest size-class, i.e. when $D$ closes to zero.)(Hara 1984; Kohyama 1991; Condit \& Sukumar 1998; Coomes et al. 2003; Muller-Landau et al. 2006b; Moore et al. 2020).

However, the deviation of observations to the mathematical prediction has been causing debates, some of which cannot be simply attributed to disturbances or the non-ideality of field data(Coomes et al.2003; Muller-Landau et al. 2006a; Muller-Landau et al.2006b; Stegen \& White 2008; Farrior et al. 2016; Zhou \& Lin 2018; Moore et al. 2020). One puzzling problem rises from the non-self-consistency of metabolic scaling theory of forest (MSTF)(Enquist et al. 2009; West et al. 2009). In this theory, power-law functions of $N$ ( $D$ 
), $G(D)$ and $M(D)$ are proposed and well fitted with convincing data cases, which are $N(D)=N{ }_{0} D$ ${ }^{-2}, G(D)=k D^{1 / 3}$, and $M(D)=2 k D^{-2 / 3}$, respectively(Enquist et al. 2009; West et al. 2009). However, putting the functional form of $G(D)$ and $M(D)$ into the equilibrium solution leads to $N(D)=N_{0} D^{-7 / 3}$ rather than $N(D)=N_{0} D^{-2}$. Lin and I (2018) have interpreted that the non-self-consistency results from the neglection of growth rate difference among size-classes in math derivations of MSTF(Zhou \& Lin 2018), which actually led to an equation of $N(D)=N_{0} \exp \left[-[?] M(D) G(D)^{-1} d D\right]$ in self-thinning. Therefore, the data were actually well fitted with a flawed theory, there is still a missing link in explaining the formation of forest size structure under demographic equilibrium state.

Here I will show how the stochasticity in tree growth rates in the same size class, which has been ignored in previous studies, affects forest size structure significantly, and solves the paradox in MSTF.

\section{Comparison of forest size structure with and without considering of growth variance}

To compare the difference between forest size structure with and without considering the stochasticity, I first assume $V, G$, and $M$ as constants (Eq. 3 and Eq. 4), since analytic solution cannot be directly given for the original form of Kolmogorov forward equation.

(3) (4) Equilibrium solutions of Eq. 3 and Eq. 4 can be easily obtained using the methods of variable separation and characteristic equation (see Supporting information), which are: Solution of Eq. 3 can be transformed as

Obviously, the exponent of $N(D)_{\text {Eq. }}$ is less negative than that of $N(D)_{\text {Eq. 3 }}$, which means in prediction of forest size structure, the decreasing rate of tree numbers with size increase will be slower in considering of a constant growth rate variance, which also implicates that the -2 power-law distribution in MSTF might be reduced from the $-7 / 3$ power due to the effect of growth rate variance.

\section{Prediction and verification for the functional form of growth variance}

In accepting $N(D)=N{ }_{0} D^{-2}, G(D)=k D^{1 / 3}$, and $M(D)=2 k D^{-2 / 3}$ in MSTF, the functional form of $V(D)$ can be expected by putting the three known functions into Kolmogorov forward equation (see Supporting information), under demographic equilibrium condition, this lead to: and:

In a prior perspective, it is basically acceptable that the variance of growth rate increases with tree size and mean growth rate, a power relationship between diameter and growth rate variance was also verified with data from Barro Colorado Island (BCI) forest(Condit et al.2012) (Fig. 1. B ).

Here I provide a statistical interpretation to the positive correlation between growth rate variance and tree size, that for $G(D)=\kappa \Delta^{\lambda}$, if growth rate variance derives from the intrinsic stochasticity in growth coefficient $k$ and exponent $\lambda$, which mean values are $\mu_{\kappa}$ and $\mu_{\lambda}$, and variances are $\sigma^{2}{ }_{k}$ and $\sigma^{2} \lambda$, then $V(D$ ) can be expressed as (see Supporting information for full derivation(Limpert et al. 2001)): (5)

In acceptable range of the parameter values based on theoretical and empirical studies, where $\mu_{\lambda}=1 / 3$ and $\sigma^{2}{ }_{\lambda}$ varies around $0.01 \sim 0.1$, functional curve of the statistical-based $V(D)$ is quite close to the power-law function with the exponential value of $4 / 3$ (Fig. 1. A ). Besides of the exponential value, the coefficient of $V(D)$, as expected to be $0.6 k$ when the coefficient of $G(D)$ is $k$, depends on the accounting time scale of $G(D)$. For instance, $G(D)$ accounted with 5-years' time interval is 5 times more than that accounted with 1-year's time interval, but variance of the former will be 25 times more than the latter, which is the square of the ratio in $G(D)$ 's magnitude changes. Hence the relative magnitude between $G(D)$ and $V$ $(D)$ will be different with the change of accounting time scale.

In estimating of forest size structure, if bin widths of size classes are at the magnitude of one to ten centimeters, as was once used in MSTF(Enquist \& Nicklas 2001), that would be diameter increments in 5- to 10-years' growth according to empirical evidences. In this premise, the estimated coefficient value of $V(D)$ based on the data from BCI forest is just close to that being expected to satisfy the -2 power-law distribution (Fig. 1. B ). However, if size classes are bound with smaller bin widths, as in later studies challenging 
MSTF(Muller-Landau et al. 2006b), the relative magnitude of $V(D)$ will be quite small to lead significant deviation of size structure from that predicted with $N(D)=N_{0} \exp \left[-[?] M(D) G(D)^{-1} d D\right] G(D)^{-1}$.

\section{Discussion}

Therefore, under particular circumstances, the inevitable variance in growth rate surely has a probability in pulling back $N(D)$ from $D^{-7 / 3}$, which determined by $G(D)$ and $M(D)$ in MSTF, to approximately $D^{-2}$ in statistics, but not precisely (Fig. 2 ). The so-called energetic equivalence among different size classes in forests(Perkins et al. 2019), is not a result of biological mechanism (i.e. considering a forest as a tree(West et al. 2009)), but a statistical coincidence. Meanwhile, since $V(D)$ is significantly affected by the accounting time scale of $G(D)$, which is reflected as the bin width of size classes in size structure estimation, it is no wonder that the estimated forest size structures deviate from the power-law distribution with the changes of growth rate, bin width selection, or estimation methods(White et al. 2008).

Although the evidences of growth rate variance, mean growth rate, mortality and forest size structure were well linked in the special case of MSTF, more generalized interpretation and quantification of their relationships are still needed. Basically, an explicit equilibrium solution to the Kolmogorov forward equation, which in the form of $N(D)=f(G(D), V(D), M(D))$, if exist, would be expected, so that foresters can get an intuitive understanding to the respective roles of $G(D), V(D)$, and $M(D)$ on forest size structure formation, and predict the large scale forest size structure from small scale inventories. Further than the static analysis on demographic equilibrium state, time dynamic analysis of forest size structure would be more challenging, especially in considering of the stochasticity in growth rate. Although ecologists believe that forests would internally tend to equilibrium in the absence of disturbance, how a forest started with any arbitrary size-density distribution finally converge to the approximately power-law distribution has never been strictly proved, mathematical approaches in stability theory may be adopted for the analysis.

However, as a posteriori function, Kolmogorov forward equation does not provide any biological inferences to forest dynamics, it simply reveals the physical truth in how growth and mortality affect individual numbers in different size classes, biological insights on specific processes in growth and mortality, e.g. size dependent growth, age dependent death, or the effects of competition, etc., require additional experimental and theoretical investigations. But without a clear understanding to the certain mathematical results, ecological phenomena may be mis-interpreted with inappropriate anticipation to biological mysteries.

\section{References}

1.

Condit, R., Chisholm, R.A. \& Hubbell, S.P. (2012). Thirty Years of Forest Census at Barro Colorado and the Importance of Immigration in Maintaining Diversity. PLOS ONE , 7, e49826.

2 .

Condit, R. \& Sukumar, R. (1998). Predicting population trends from size distributions: a direct test in a tropical tree community.American Naturalist , 152, 495-509.

3.

Coomes, D.A., Duncan, R.P., Allen, R.B. \& Truscott, J. (2003). Disturbances prevent stem size-density distributions in natural forests from following scaling relationships. Ecology Letters , 6, 980-989.

4 .

Enquist, B.J. \& Nicklas, K.J. (2001). Invariant scaling relations across tree-dominated communities. Nature , 410, 655-660.

5 .

Enquist, B.J., West, G.B. \& Brown, J.H. (2009). Extensions and evaluations of a general quantitative theory of forest structure and dynamics. Proceedings of the National Academy of Sciences of the United States of 
America , 106, 7046-7051.

6.

Farrior, C.E., Bohlman, S.A., Hubbell, S. \& Pacala, S.W. (2016). Dominance of the suppressed: Power-law size structure in tropical forests. Science, 351, 155-157.

7 .

Hara, T. (1984). A Stochastic Model and the Moment Dynamics of the Growth and Size Distribution in Plant Populations. Journal of Theoretical Biology , 109, 173-190.

8.

Kohyama, T. (1991). Simulating stationary size distribution of trees in rain forests. Annals of Botany , 68, 173-180.

9 .

Limpert, E., Stahel, W.A. \& Abbt, M. (2001). Log-normal Distributions across the Sciences: Keys and Clues: On the charms of statistics, and how mechanical models resembling gambling machines offer a link to a handy way to characterize log-normal distributions, which can provide deeper insight into variability and probability — normal or log-normal: That is the question. BioScience , 51, 341-352.

10.

Moore, J.R., Argles, A.P.K., Zhu, K., Huntingford, C. \& Cox, P.M. (2020). Validation of demographic equilibrium theory against tree-size distributions and biomass density in Amazonia. Biogeosciences , 17, 1013-1032.

11.

Muller-Landau, H.C., Condit, R.S., Chave, J., Thomas, S.C., Bohlman, S.A., Bunyavejchewin, S. et al. (2006a). Testing metabolic ecology theory for allometric scaling of tree size, growth and mortality in tropical forests. Ecology Letters, 9, 575-588.

12.

Muller-Landau, H.C., Condit, R.S., Harms, K.E., Marks, C.O., Thomas, S.C., Bunyavejchewin, S. et al. (2006b). Comparing tropical forest tree size distributions with the predictions of metabolic ecology and equilibrium models. Ecol Lett, 9, 589-602.

13.

Perkins, D.M., Perna, A., Adrian, R., Cermeno, P., Gaedke, U., Huete-Ortega, M. et al. (2019). Energetic equivalence underpins the size structure of tree and phytoplankton communities. Nature communications, 10,255 .

14.

Stegen, J.C. \& White, E.P. (2008). On the relationship between mass and diameter distributions in tree communities. Ecol Lett, 11, 1287-1293.

15.

Von Foerster, H. (1959). Some remarks on changing populations. In: The Kinetics of Cellular Proliferation (ed. Stohlman, F. Jr). Grune and Stratton, New York, NY,. 382-407.

16. 
West, G.B., Enquist, B.J. \& Brown, J.H. (2009). A general quantitative theory of forest structure and dynamics. Proceedings of the National Academy of Sciences of the United States of America, 106, 70407045 .

17.

White, E.P., Enquist, B.J. \& Green, J.L. (2008). ON ESTIMATING THE EXPONENT OF POWER-LAW FREQUENCY DISTRIBUTIONS. Ecology , 89, 905-912.

18.

Zhou, J. \& Lin, G. (2018). Will Forest Size Structure Follow the -2 Power-Law Distribution under Ideal Demographic Equilibrium State? J Theor Biol .

\section{Acknowledgements}

The study is supported by National Key Research Development Program of China (2017YFA0605101)

\section{Figures and Legends}

\section{Hosted file}

image11.emf available at https://authorea.com/users/314145/articles/444533-stochasticity-intree-growth-closes-the-chain-of-evidence-in-forest-structural-dynamics

Figure 1. The relationship between tree size and growth rate variance in theoretical prediction (A) and data verification (B). The curve of variance derived from statistical rules (red line in subplot A) is based on Eq. 5 , parameter values were set as $\mu_{\lambda}=1 / 3, \sigma_{\lambda}^{2}=0.05, \mu_{\kappa}=0.25, \sigma_{k}^{2}=0.25$.

\section{Hosted file}

image12.emf available at https://authorea.com/users/314145/articles/444533-stochasticity-intree-growth-closes-the-chain-of-evidence-in-forest-structural-dynamics

Figure 2 . Estimated size-frequency distributions of BCI forest under different bin-widths of size classes. To verify the effect of growth rate variance, size classes were bound with the estimated mean diameter increments of the corresponding sizes, in approximately 5-years (blue dots) and 1-month (red dots). This way of data grouping is different with the linear- or log-even size classes, which were used previously and would lead to a decreasing exponent with the magnitude increase of bin-width due to statistical bias(White et al. 2008). 